\title{
CLASSIFICATION OF STARS WITH DIFFERENT CHEMICAL CONSTITUTION
}

\author{
D. CHALONGE and L. DIVAN \\ Institut d'Astrophysique, Paris, France
}

\begin{abstract}
The possibility of obtaining a segregation between stars of different chemical constitution using the BCD classification is reviewed.
\end{abstract}

It is now well known that large differences in chemical composition do exist between the various families of stars, but the MK classification seems to be reserved to the so-called 'normal stars' having a chemical composition more or less similar to the solar one: neither the 'metallic line stars' nor the stars of population II which have chemical compositions very different, and very different from the one of the normal stars, can find a place in it.

After the pioneer work of Unsöld on $\tau$ Scorpii, the accurate analysis of the composition of a number of normal stars, performed in various observatories, has shown that rather small but nevertheless conspicuous differences of chemical constitution exist between them.

Thus, since large differences of constitution prevent a classification, one may wonder whether the small differences observed between the normal stars do not bring certain distorsions in their classification.

The conclusion is that it is desirable to improve the actual system of classification in order to open it to all kinds of chemical composition. This would require the introduction of, at least, one more parameter to take into account the behaviour of stars with different compositions.

During a symposium on spectral classification held in Paris in 1953, we described such a three-dimensional system of stellar classification and gave its first results: they seem to be in good agreement with those of the MK classification for the normal stars and proved that this new system was opened to stars with composition so different as the metallic line stars and the subdwarfs.

Since that time we have applied the method to the classification of several hundreds of stars of different types in order to give a stronger foundation to this new system, and we have now enough material to confirm and to complete our first conclusions. Some of these new results have been presented during Symposium no. 24 held in 1966 at Saltsjöbaden (the corresponding paper will be referred to as number I), and, with more details, last year in a paper published in Astron. Astrophys. (which will be referred to as number II).

So we give here only a short account on the method and on the actual situation. In this system of classification every star is characterized by the numerical values of three quantitative parameters describing the distribution of energy in the continuum of the 
star. Our parameters concern a layer of the star somewhat deeper than the layers used in the MK classification but unfortunately this method requires, for the measurement of the values of the parameters, that the star to be classified presents, all along its spectrum, 'windows' permitting to reach these deeper layers.

This condition is fulfilled only in the early types, O, B, A, F, so that our classification is restricted to these types, that is, to the more massive and younger stars. The three parameters, all pertaining to the continuum, are the spectrophotometric gradient $\phi_{b}$ corresponding to the colour temperature of the continuum in the blue-violet region of the spectrum, the Balmer discontinuity $D$ and a wavelength $\lambda_{1}$ characteristic of the real position of the discontinuity in the spectrum.

The first parameter is very sensitive to the interstellar absorption, so that its real value can be directly determined only for the nearby stars.

But the two other parameters are not affected by this absorption: they give, without any correction, the value of two intrinsic features of the star and the main advantages of the present system of classification are direct consequences from this fact. When we know the intrinsic values of the three parameters for a star we may represent it by a point with the co-ordinates $\phi_{b}, D$ and $\lambda_{1}$ in a three-dimensional system of co-ordinates. The representative points of the normal stars fall very near to a surface which is represented in Figure 1 (paper I): the different spectral types are distributed in well-determined areas on the surface. Figures 2 and 3 of paper I show respectively that the metallic-line stars and the population II stars, which have a different chemical composition, are represented by points situated well out of the surface and on both sides of it. The few blue stars of the halo represented on Figure 3 (paper I), which probably belong also to population II, would eventually not lie far from the surface if their gradient was corrected from a small interstellar absorption.

If we return to the normal stars, those who have been found somewhat more metal rich or more metal poor lie in the immediate vicinity of the surface, on the right side for the first, on the left for the second.

Figure 1 of the present paper represents the same results in another system of co-ordinates: every star is represented by a point with $\phi_{b}$ as ordinate, and as abscissa a variable $s$ giving a quantitative measure of the spectral type; the definition of $s$ has been given in paper II. The few not reddened $\mathrm{O}$ and $\mathrm{B}$ stars are distributed along a very regular line but the late $A$ and the $F$ stars of Figure 1, which are all nearby stars with no interstellar reddening, show a significant scatter and lie between the two dotted lines. An average line has been drawn between them and it may be seen that the metal-rich star $\beta$ Vir falls above the average line, and the metal-poor $\chi$ Dra, $\xi$ Peg, $\sigma$ Boo fall below. The majority of the Hyades are situated above the average line and also the members of the galactic cluster in Coma: this suggests that they are somewhat more metal-rich than the Sun, a result which has been found by various observers for later members of the two clusters. The average line would correspond to stars having exactly the same composition as the Sun.

The difference in the distribution of the $B$ and of the F stars is probably due to the 


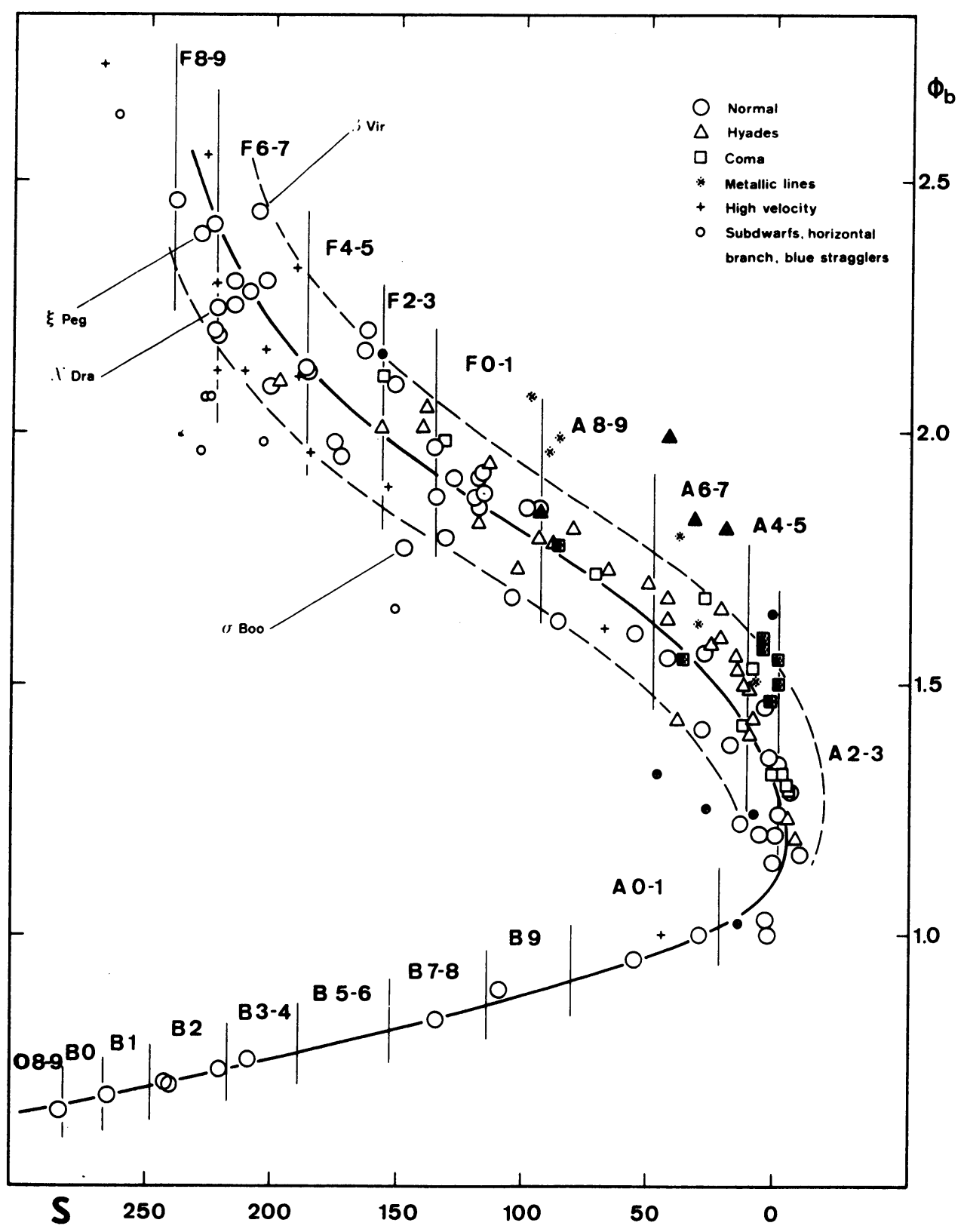

fact that the $\mathrm{F}$ belong (because of their relatively slow evolution) to several generations of stars with various compositions and the $\mathrm{B}$ only to the last one, a rapid evolution having brought the others to other parts of the diagram.

The metallic-line stars, fall in general more above the average line than the above- 
mentioned metal-rich stars and the subdwarfs and horizontal branch stars lie much below this line; the high velocity stars lie also below the average line; a part of them belong to the population II.

It thus appears that our classification agrees with the MK one only for stars lying on the 'average line' of Figure 1; and these stars should be considered as the real 'normal stars'. For the others the figure suggests that they are either somewhat more metal-rich or metal-poor than the normal stars, a result which cannot be expressed by the MK parameters alone and would require the use of a third parameter.

\section{References}

Chalonge, D.: 1955, Principes Fondamentaux de Classification Stellaire, Colloques Internationaux du Centre National de la Recherche Scientifique, Paris, p. 55.

Chalonge, D.: 1966, in K. Lodén, L. O. Lodén and U. Sinnerstad (eds.), IAU Symp. 24, 77, Academic Press, London.

Chalonge, D. and Divan, L.: 1973, Astron. Astrophys. 23, 69.

\section{DISCUSSION}

Cayrel: Are the iso-metal-content lines you found for the Hyades and Coma the same as those in the Strömgren photometry?

Chalonge: We have not studied the same members of the two clusters as those observed in the Strömgren photometry: we have considered their earliest members and, in the Strömgren spectroscopy, latest members have been observed But both works lead to the same conclusions: these two clusters are somewhat more metal-rich than the Sun. We have not given iso-metal-content lines. 